1 running title: An application for aiding COVID-19 diagnosis

2 Development and utilization of an intelligent application for aiding COVID-19

3 diagnosis

4 Zirui Meng ${ }^{1, a}$, Minjin Wang ${ }^{1, a}$, Huan Song ${ }^{2, a}$, Shuo Guo ${ }^{1}$, Yanbing Zhou ${ }^{1}$, Weimin Li $^{3}$,

5 Yongzhao Zhou ${ }^{3}$, Mengjiao $\mathrm{Li}^{1}$, Xingbo Song ${ }^{1}$, Yi Zhou ${ }^{1}$, Qingfeng $\mathrm{Li}^{4}, \mathrm{Xiaojun} \mathrm{Lu}^{1}$,

$6 \quad$ Binwu Ying, ${ }^{1, *}$

$7{ }^{1}$ Department of Laboratory Medicine, West China Hospital, Sichuan University,

8 Chengdu, Sichuan Province, China

$9{ }^{2}$ West China Biomedical Big Data Center, West China Hospital, Sichuan University,

10 Chengdu, Sichuan Province, China

$11{ }^{3}$ Department of Respiratory and critical care medicine, West China Hospital, Sichuan

12 University, Chengdu, Sichuan Province, China

$13{ }^{4}$ Department of Clinical Laboratory, Public Health Clinical Center of Chengdu,

14 Chengdu, Sichuan Province, China.

$15{ }^{a}$ Zirui Meng, Minjin Wang, Huan Song. contributed equally to this work.

16 Correspondence author: Binwu Ying. No.37 Guoxue Alley, West China Hospital,

17 Sichuan University, Chengdu 610041, Sichuan Province, P. R China.

Tel:86-028-85422751

19 Fax:86-028-85422751

Email:binwuying@126.com 


\section{Development and utilization of an intelligent application for aiding COVID-19}

\section{diagnosis}

\section{ABSTRACT}

Background: COVID-19 has been spreading globally since emergence, but the diagnostic resources are relatively insufficient.

Results: In order to effectively relieve the resource deficiency of diagnosing COVID-19, we developed a machine learning-based diagnosis model on basis of laboratory examinations indicators from a total of 620 samples, and subsequently implemented it as a COVID-19 diagnosis aid APP to facilitate promotion.

Conclusions: External validation showed satisfiable model prediction performance (i.e., the positive predictive value and negative predictive value was $86.35 \%$ and $84.62 \%$, respectively), which guarantees the promising use of this tool for extensive screening.

Keywords: Corona Virus Disease 2019; diagnostic; machine learning; laboratory testing; Application.

\section{BACKGROUND}

Since the outbreak of Corona Virus Disease 2019(COVID-19) in Wuhan, in December 2019, the epidemic has spread rapidly all over the world, which consequently brought great challenges to global public health(1). As of March 14, a total of 142,539 cases had been confirmed worldwide, with many more requiring tests(2). Currently, accurate nucleic acid detection plays a pivotal role in diagnosis and prevention of 
COVID-19, and reverse transcription polymerase chain reaction is the main method for nucleic acid detection(3). However, it is not feasible to use this time-and labor-consuming approach for screening a large growing number of suspected patients and asymptomatic infected patients(4). Rapid and universal screening method is essential to save medical resources, improve diagnosis efficiency and avoid cross-infection.

Taking advantage of the emerging machine learning technique, which enables analysis of the existing multidimensional data by applying appropriate algorithms for feature expression and classification, we have potential to improve the accuracy of diagnosis. For instance, in a recent study published in Nature, deep learning-based statistical model was used to improve breast cancer treatment and survival through identifying breast cancer patients with a high risk of long-term recurrence(5). In this study, we analyzed a variety of basic laboratory examinations indicators that related to host reactions to generate a free COVID-19 diagnosis aid APP. Then, the accuracy of this app was validated in an independent cohort(6).

\section{METHODS}

\section{Patients and information collection}

We have complied with all relevant ethical regulations for work with human subjects and the studies were approved by the Clinical Trials and Biomedical Ethics Committee of West China Hospital, Sichuan University. Through the West China hospital, a regional medical center, we collected the information of confirmed COVID-19 cases from various regional medical institutions, between 20th December 
67

2019 and 10th February 2020. Diagnostic criteria were: RT-PCR of respiratory or blood samples was positive for SARS-CoV-2 (Severe Acute Respiratory Syndrome Coronavirus 2) nucleic acid; or viral gene sequencing of respiratory or blood sample was highly homologous with SARS-CoV-2. Controls were patients collected in the same site and the same time period, who were free of COVID-19 but had a diagnosis of viral pneumonia. Results of laboratory examinations on admission were collected which included age, gender and 35 indicators of whole blood count, coagulation test and biochemical examination. In addition, the independent validation cohort consisted of samples collected prospectively from one single medical center, using similar data collection strategy.

\section{Feature selection and model construction}

Factors under consideration are age, sex and indicators of basic laboratory examinations(see Table 1). Using 70\% samples from the derivation cohort (i.e., the training set), we first performed feature selection using Least Absolute Shrinkage and Selection Operator (LASSO) to select the valuable indicators of COVID-19. The main idea of LASSO for feature selection is to construct a first-order penalty function, which can shrink the regression coefficient (b) of each variable within a certain range, and eliminate the feature with a coefficient of 0 , and finally obtain an optimally refined model(7). The penalty terms for LASSO are: $\operatorname{sum}(\operatorname{abs}(b))<=t$. Then these indicators were used to construct a primitive model by multivariate logistic regression. The parameters were further adjusted through internal validation (i.e., the other $30 \%$ samples, the testing set) to obtain the optimized final model. 
89

90

91

92

\section{Evaluation}

In external validation cohort, we compared the predicted results, with the follow-up results to evaluate the diagnostic validity by positive predictive values, and negative predictive values. Finally, to facilitate further use in clinic setting, all model parameters and code were encapsulated as a visual application(APP), COVID-19 Diagnosis Aid APP.

\section{Statistical analysis}

Continuous variables are represented by the median (upper and lower quartiles). Categorical variables are expressed in terms of frequency. LASSO algorithm was used for feature selection and the model was constructed by multivariate logistic regression. The diagnostic performance of the model was assessed by AUC. Calibration curves and Hosmer-Lemeshow test were used to evaluate the degree of overestimation or underestimation of the model. DCA were used to measure the net clinical benefits. The LASSO algorithm was performed by "glmmet" package. The logistic regression model was constructed by "glm" package. All statistical analyses were completed using R 3.5.0 version.

\section{RESULTS}

\section{Participants and Clinical Characteristics}

A total of 620 samples were included in the derivation cohort, among which 431 samples (211 COVID-19 vs. 220 Control) were in the training set and 189 samples (91 COVID-19 vs. 98 Control) were in testing set by simple randomization. The frequency of COVID-19 in the training set (48.96\%) was not significantly different 
111 from that in the testing set $(48.14 \%)$. We included 145 samples in the independent

112 validation cohort.

113 Model development and evaluation

114 Through Lasso regression screening and Multivariate logistic regression, 9 115 representative variables (age, Activated Partial Thromboplastin Time, Red Blood Cell

116 Distribution Width-SD, Uric Acid, Triglyceride, Serum Potassium, Albumin/globulin, 117 3-Hydroxybutyrate, Serum Calcium) with good identification value were selected and 118 constructed an optimized diagnostic model(see Figure 1). According to the suggestive 119 information from the model, among 145 samples that used for validation, 80 samples 120 were estimated as COVID-19 and 65 samples were considered COVID-19-free. 121 Compared with the status determined by nucleic acid detection (the golden standard), 122 we found 69 real COVID-19 samples were correctly identified by the app $(69 / 80$, 123 positive predictive value $=86.25 \%$ ), while 55 , out of the 65 COVID-19-free samples, 124 were confirmed to be negative $(55 / 65$, negative predictive value $=84.62 \%)$. The area 125 under curve (AUC) of the model were 0.890 and 0.872 in the testing set and 126 independent validation cohort, respectively(see Figure 2)(8). The calibration curve 127 performed well and Hosmer-Lemeshow test results $\mathrm{P}$ value was much greater than 0.05(see Figure 3). The DCA quantitatively demonstrated high clinical net benefit 129 over the entire probability threshold(see Figure 4)(9).

\section{Construction of application}

131 The model was encapsulated as an APP, COVID-19 Diagnosis Aid APP. The risk of 132 COVID-19 can be obtained by inputting the required indicators into the APP. It will 
133

134

135

136

137 be online in the android store and IOS APP Store soon for facilitating the further application(see Figure 5).

\section{DISCUSSION}

According to the latest epidemiological information of the World Health Organization(WHO), SARS-CoV-2 is spreading worldwide with an intensifying situation, and many countries including South Korea, Italy, and the United States have experienced outbreaks(10). Timely diagnosis, isolation and treatment are critical to control the global spread of COVID-19. However, nucleic acid testing is not always feasible or fully affordable in all regions. We therefore need more ways to realize the early and accurate community control and screening of suspicious people. To our knowledge, this is the first COVID-19 Diagnosis Aid APP based on laboratory tests for extensive screening. It has good accuracy and stability, which can help medical institutions to predict patients' situation in advance and accurately invest the limited healthcare resources, so as to effectively control the spread of the epidemic.

Based on the optimized diagnostic model developed in current study, users can obtain the probability of COVID-19 according to the simple, easily available and reliable indicators from laboratory examinations. Given satisfiable model prediction performance (i.e., AUC was 0.872; positive predictive value and negative predictive value was reach $86.25 \%$ and $84.62 \%$, respectively in independent validation cohort), the application of this app can provide reliable suggestion for further assessment of the index person, which has potential to reduce anxiety of public, as well as unnecessary hospital visit and nucleic acid testing. 
155 It is undeniable that nucleic acid detection is one of the most reliable standards for the

156 diagnosis of COVID-19, but we should also be aware of its shortcomings and

157 deficiencies in practice. Nucleic acid testing requires professional technical platforms

158 and physicians, and false negative results due to multiple reasons cannot be

159 completely avoided(11). Instead, laboratory indicators that identified in our study are

160 accessible, and can be widely used in community hospitals by family doctors to

161 complete the early stage of suspected population screening.

162 On the other hand, based on the technological revolution brought by the Internet, this

APP software installed on personal mobile can not only help to discover COVID-19

in the most convenient way, but also timely monitor dynamic trends of the prevalence

of the contagious disease through data transmission to the Disease Control Center or

professional medical institution $(12,13)$. This provides real-time data with predictive

167 value, which can be reliable basis for more accurate health and epidemic prevention

possibility of extended scope of application. For example, we have added the

173 pre-connection between individuals and medical centers, which may benefit patients

174 by avoiding unnecessary hospital visits or shorten waiting time spend before 175 admission (14). 
177 COVID-19 Diagnosis Aid APP can efficiently and accurately calculate the infection

178 probability through simple and easily-obtained laboratory examination results. It will

179 promote the screening of a large number of suspected people, save limited medical

180 resources, optimize the diagnosis process, and it can constantly learn, adapt and

181 upgrade, which are vital to control the global spread of COVID-19.

\section{Ethics approval and consent to participate}

184 The protocol of this study was approved by the West China Hospital, Sichuan

185 University Medical Ethics Committee and conformed to the principles of the 186 Declaration of Helsinki.

187 The registry number for clinical trial

188 ChiCTR2000030542

189 Consent for publication

190 Not applicable

191 Data Availability Statement

192 The datasets used and analysed during the current study are available from the 193 corresponding author on reasonable request.

194 Competing interests

195 The authors declare that they have no competing interests

196 Funding

197 This study was supported by Science and Technology Department of Sichuan 198 Province "2020YFS0004" and Science and Technology Project of West China 
199

200

201

202

203

204

205

206

207

208

209

210

211

212

213

214

215

216

217

218

219

220

Hospital "HX-2019-nCov-066"

\section{Authors' contributions}

Zirui Meng analyzed the data and was a major contributor in writing the manuscript.

Minjin Wang designed the APP framework and was a major contributor in writing the manuscript. Huan Song analyzed the data and was a major contributor in writing the manuscript. Shuo Guo analyzed the data. Yanbing Zhou analyzed the data. Weimin Li analyzed patients' medical records. Yongzhao Zhou analyzed patients' medical records. Mengjiao Li collected the data. Xingbo Song verified the performance of the app. Yi Zhou verified the performance of the app. Qingfeng Li collected the data. Xiaojun Lu designed the functions of the app. Binwu Ying proposed research ideas and design research programs. All authors read and approved the final manuscript.

\section{Acknowledgements}

The authors thank Beijing Zhongben Tech.co,.Ltd. for their technical contributions to this study.

\section{REFERENCE}

1. Z W, JM M. Characteristics of and Important Lessons From the Coronavirus Disease 2019 (COVID-19) Outbreak in China: Summary of a Report of $72 \square 314$ Cases From the Chinese Center for Disease Control and Prevention. JAMA. 2020.

2. Data as reported by national authorities by 10 AM CET 14 March 2020 [Available

from: https://www.who.int/docs/default-source/coronaviruse/situation-reports/20200314-sitr ep-54-covid-19.pdf?sfvrsn=dcd46351_6. 
221

222

223

224

225

226

227

228

229

230

231

232

3. VM C, O L, M K, R M, A M, DKW C, et al. Detection of 2019 novel coronavirus (2019-nCoV) by real-time RT-PCR. Euro surveillance : bulletin Europeen sur les maladies transmissibles $=$ European communicable disease bulletin. 2020;25(3).

4. T K, SM J, NM L, R K, K H, T M, et al. Communicating the Risk of Death from Novel Coronavirus Disease (COVID-19). Journal of clinical medicine. 2020;9(2).

5. OM R, SJ S, JA S, SF C, JL C-J, M C, et al. Dynamics of breast-cancer relapse reveal late-recurring ER-positive genomic subgroups. Nature. 2019;567(7748):399-404.

6. F S, N S, F S, Z Z, J S, H L, et al. Emerging Coronavirus 2019-nCoV Pneumonia. Radiology. 2020:200274.

7. Tibshirani R. The lasso method for variable selection in the cox model. Stat Med. 1997;16(4):385-95.

8. Muntner P, Colantonio LD, Cushman M, Goff DC, Jr., Howard G, Howard VJ, et al. Validation of the atherosclerotic cardiovascular disease Pooled Cohort risk equations. JAMA. 2014;311(14):1406-15.

9. Vickers AJ, Elkin EB. Decision curve analysis: A novel method for evaluating prediction models. Med Decis Making. 2006;26(6):565-74.

10. S BS, P R, Y S, A M, C C, A S, et al. First cases of coronavirus disease 2019 (COVID-19) in France: surveillance, investigations and control measures, January 2020. Euro surveillance : bulletin Europeen sur les maladies transmissibles = European communicable disease bulletin. 2020;25(6).

11. T A, Z Y, H H, C Z, C C, W L, et al. Correlation of Chest CT and RT-PCR Testing 
medRxiv preprint doi: https://doi.org/10.1101/2020.03.18.20035816; this version posted March 21, 2020. The copyright holder for this preprint (which was not certified by peer review) is the author/funder, who has granted medRxiv a license to display the preprint in perpetuity. All rights reserved. No reuse allowed without permission.

243 in Coronavirus Disease 2019 (COVID-19) in China: A Report of 1014 Cases.

244 Radiology. 2020:200642.

245 12. CS W, MR T, J B, TP M-T, K H, D P, et al. Taking connected mobile-health

246 diagnostics of infectious diseases to the field. Nature. 2019;566(7745):467-74.

247 13. EV G, A S, ND C. Using the Internet to identify infectious-disease outbreaks. The

248 New England journal of medicine. 2004;351(24):2558-9.

249 14. JT W, K L, GM L. Nowcasting and forecasting the potential domestic and 250 international spread of the $2019-\mathrm{nCoV}$ outbreak originating in Wuhan, China: a 251 modelling study. Lancet (London, England). 2020. 
medRxiv preprint doi: https://doi.org/10.1101/2020.03.18.20035816; this version posted March 21, 2020. The copyright holder for this preprint (which was not certified by peer review) is the author/funder, who has granted medRxiv a license to display the preprint in perpetuity.

All rights reserved. No reuse allowed without permission.

265 Table 1. Candidate of the model.

Derivation cohort

Control

SEX

AGE

PT

INR

APTT

$28.18(29.75,35.68)$

$17.10(18.00,19.13)$

$\mathrm{RBC}$

$3.13(3.76,4.13)$

HGB

$93.25(110.50,126.50)$

HCT

$0.29(0.35,0.39)$

88.13(92.20,96.30)

$29.00(29.90,31.20)$

$\mathrm{MCH}$

MCHC

$317.00(325.00,335.25)$

RDW-SD

$44.58(47.45,51.13)$

RDW-CV $\quad 13.40(14.10,15.33)$

PLT

$135.75(186.00,230.75)$

WBC

$5.73(7.39,9.02)$

TBIL

$6.20(9.75,15.18)$

DBIL
COVID-19

female $(31.07 \%)$

$46(55,73)$

10.7(11.8, 13.4)

$0.94(1.04,1.16)$

$25.60(27.90,31.40)$

$16.40(17.20,18.10)$

$3.66(4.25,4.76)$

$111.00(129.00,148.00)$

$0.35(0.38,0.44)$

$88.50(91.50,95.00)$

$28.90(30.70,32.10)$

$320.00(334.00,345.00)$

$41.90(44.50,49.80)$

$12.80(13.60,15.10)$

$107.00(147.00,202.00)$

$6.52(9.59,14.89)$

$8.50(12.20,17.50)$

$3.70(5.70,7.50)$

Validation cohort

Control

COVID-19

female(31.21\%)

$66(76,84)$

$11.1(12,13.7)$

$0.97(1.06,1.20)$

$27.38(30.10,35.43)$

17.20(18.20,19.60)

$3.10(3.68,4.15)$

$91.75(110.00,123.00)$

$0.29(0.34,0.38)$

$88.90(93.25,97.20)$

$28.90(30.00,31.30)$

$314.00(324.00,332.00)$

$45.08(49.20,54.10)$

$13.70(14.60,15.90)$

$130.00(203.50,276.25)$

$5.87(8.02,11.04)$

$6.90(9.65,14.48)$

$2.90(4.40,7.13)$
$126.75(203.50,277.75)$

5.22(7.42,11.09)

female $(30.56 \%)$

$48(58,67)$

11(11.7,12.7)

$0.96(1.02,1.12)$

$25.68(28.25,32.20)$

$16.98(17.90,19.10)$

$3.47(3.98,4.44)$

$103.00(119.00,135.00)$

$0.32(0.36,0.40)$

$88.40(91.65,95.53)$

$29.10(0.40,31.70)$

$318.00(328.00,339.00)$

$42.65(46.20,50.25)$

$13.18(13.90,14.90)$

7.60(10.30,14.40)

$3.30(4.60,7.23)$ 
medRxiv preprint doi: https://doi.org/10.1101/2020.03.18.20035816; this version posted March 21, 2020. The copyright holder for this preprint (which was not certified by peer review) is the author/funder, who has granted medRxiv a license to display the preprint in perpetuity.

All rights reserved. No reuse allowed without permission.

\begin{tabular}{|c|c|c|c|c|}
\hline IBIL & $3.78(4.95,6.98)$ & $4.00(6.10,9.10)$ & $3.30(4.60,7.10)$ & $3.40(5.10,7.50)$ \\
\hline ALT & $12.00(19.00,30.25)$ & $18.00(27.00,55.00)$ & $13.00(21.00,37.00)$ & $19.00(31.50,57.00)$ \\
\hline AST & $18.75(23.00,36.00)$ & $22.00(36.00,68.00)$ & $19.00(25.50,39.25)$ & $21.00(33.00,55.00)$ \\
\hline $\mathrm{TP}$ & $53.90(58.85,68.13)$ & $60.80(65.90,72.10)$ & $56.48(62.05,68.53)$ & $55.90(60.85,66.33)$ \\
\hline ALB & $32.70(36.10,41.65)$ & $30.70(34.10,39.00)$ & $31.30(34.75,38.53)$ & $29.90(32.95,37.23)$ \\
\hline GLB & $19.43(23.80,28.45)$ & $27.50(31.80,35.90)$ & $22.50(26.50,30.93)$ & $23.38(27.00,31.60)$ \\
\hline $\mathrm{A} / \mathrm{G}$ & $1.33(1.51,1.91)$ & $0.90(1.11,1.32)$ & $1.11(1.35,1.61)$ & $1.03(1.22,1.46)$ \\
\hline GLU & $4.92(6.00,7.64)$ & $6.31(7.33,9.67)$ & $5.49(7.20,9.40)$ & $5.10(6.24,8.40)$ \\
\hline UREA & $4.60(6.05,8.25)$ & $4.20(6.20,10.40)$ & $4.58(6.69,9.43)$ & $3.40(4.73,7.00)$ \\
\hline CREA & $50.50(60.50,103.50)$ & $60.00(75.00,94.00)$ & $50.00(65.50,90.00)$ & $51.00(61.00,77.00)$ \\
\hline eGFR & $60.87(89.49,97.73)$ & $66.14(96.38,104.66)$ & $63.45(86.14,100.87)$ & $87.21(101.88,112.20)$ \\
\hline Cys-C & $0.90(1.06,1.69)$ & $0.86(1.06,1.42)$ & $0.92(1.16,1.68)$ & $0.82(0.96,1.23)$ \\
\hline URIC & $154.75(219.00,291.25)$ & $181.00(281.00,366.00)$ & $133.75(200.00,287.25)$ & $138.00(192.00,281.00)$ \\
\hline TG & $0.71(0.93,1.23)$ & $0.98(1.17,1.80)$ & $0.76(1.03,1.34)$ & $0.94(1.30,1.82)$ \\
\hline $\mathrm{CK}$ & $41.75(56.00,86.75)$ & $39.00(87.00,204.00)$ & $29.00(49.50,84.25)$ & $36.00(62.00,125.25)$ \\
\hline NA & $135.30(139.20,141.83)$ & $131.40(135.50,139.10)$ & $135.95(139.70,142.60)$ & $135.80(139.15,141.70)$ \\
\hline $\mathrm{K}$ & $3.72(4.08,4.38)$ & $3.32(3.76,4.09)$ & $3.73(4.08,4.51)$ & $3.57(3.96,4.38)$ \\
\hline$\beta$-HBA & $0.07(0.12,0.23)$ & $0.08(0.23,0.38)$ & $0.07(0.11,0.22)$ & $0.06(0.12,0.30)$ \\
\hline $\mathrm{CA}$ & $2.06(2.15,2.21)$ & $2.01(2.11,2.23)$ & $2.02(2.14,2.24)$ & $1.97(2.07,2.17)$ \\
\hline
\end{tabular}

266 Data are presented as n (\%) for categorical variables and as median (interquartile range) for continuous variables.

267 PT, prothrombin time; INR, International Normalized Ratio; APTT, activated partial thromboplastin time; TT,

268 thromboplastin time; RBC, red blood cell; HGB, hemoglobin; HCT, hematocrit; MCV, Mean Corpuscular Volume; 
medRxiv preprint doi: https://doi.org/10.1101/2020.03.18.20035816; this version posted March 21, 2020. The copyright holder for this preprint (which was not certified by peer review) is the author/funder, who has granted medRxiv a license to display the preprint in perpetuity.

All rights reserved. No reuse allowed without permission.

MCH, Mean Corpuscular Hemoglobin; MCHC, Mean Corpuscular Hemoglobin Concentration; RDW-SD, Red Blood Cell Distribution Width-SD; RDW-CV, Red Blood Cell Distribution Width-CV; PLT, Platelets; WBC, white blood cell; TBIL, total Bilirubin; DBIL, direct bilirubin; IBIL, indirect bilirubin; ALT, alanine aminotransferase; AST, glutamic oxaloacetic transaminase; TP, Total Protein; Alb, albumin; ALB, albumin; GLB, globulin ratio; A/G, albumin globulin ratio; GLU, glucose; CREA, serum;eGFR, estimated Glomerular Filtration Rate; Cys-C, cystatin; URIC, uric acid; TG, Triglyceride; CHOL, cholesterol; CK, creatine kinase; NA, serum natrium; K, Serum Potassium; $\beta$-HBA, 3-Hydroxybutyrate; CA, Serum Calcium;

\section{Figure legends}

Figure.1(a) Coefficient diagram of LASSO variables. Each curve in the figure represents the trajectory of the coefficient of an independent variable. The ordinate is the value of the coefficient. The lower abscissa, $\lambda$, is the parameter that controls the severity of the penalty. The upper abscissa is the number of non-zero coefficients in the model under the penalty parameter. (b) Adjustment parameters in the LASSO

model. The lambda is screened by 10 folds cross-validation. A dashed vertical line is drawn at one standard error (1-SE standard) of the minimum and minimum standards. Lambda.1se corresponds to a model with good performance but the fewest number of arguments.

Figure 2. Receiver operator characteristic curve. The AUC were 0.890 and 0.872 in derivation and validation cohort respectively.

Figure 3. Calibration curves. The $45^{\circ}$ shaded line represents the ideal prediction and the prediction probability is consistent with the actual observation probability. The 
291 Solid line represents the actual prediction of the model. The stapled histogram on the

292 bottom line represents the distribution of patients' predicted probability. Abbreviations:

293 Dxy $=$ Somer's D rank correlation, R2 = Nagelkerke-Cox-Snell-Maddala- Magee

294 R-squared index, D = Discrimination index, $U$ = Unreliability index, $\mathrm{Q}=$ Quality

295 index, Emax = maximum absolute difference in predicted and calibrated propabilities,

296 S:z = Spiegelhalter Z-Test, S:p = two-tailed p-value of the Spiegelhalter Z-test.

297 Figure 4. Decision curve analysis. The horizontal axis is the threshold probability of occurrence of COVID-19. The vertical axis shows the clinical benefits that patients may gain or lose using the APP. Dotted line: prediction model. Solid line: all patients were COVID-19 . Horizontal line: all patients were not COVID-19. 


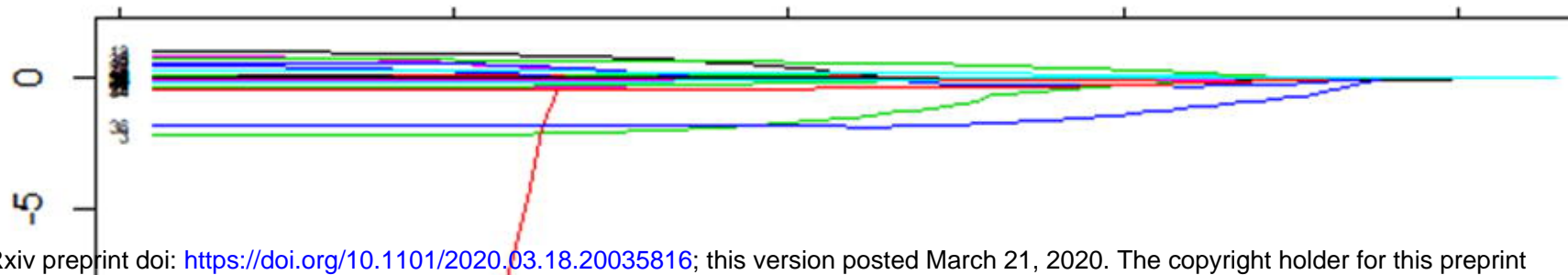

medRxiv preprint doi: https://doi.org/10.1101/2020.03.18.20035816; this version posted March 21, 2020. The copyright holder for this preprint (which was not certified by peer review) is the author/funder, who has granted medRxiv a license to display the preprint in perpetuity.

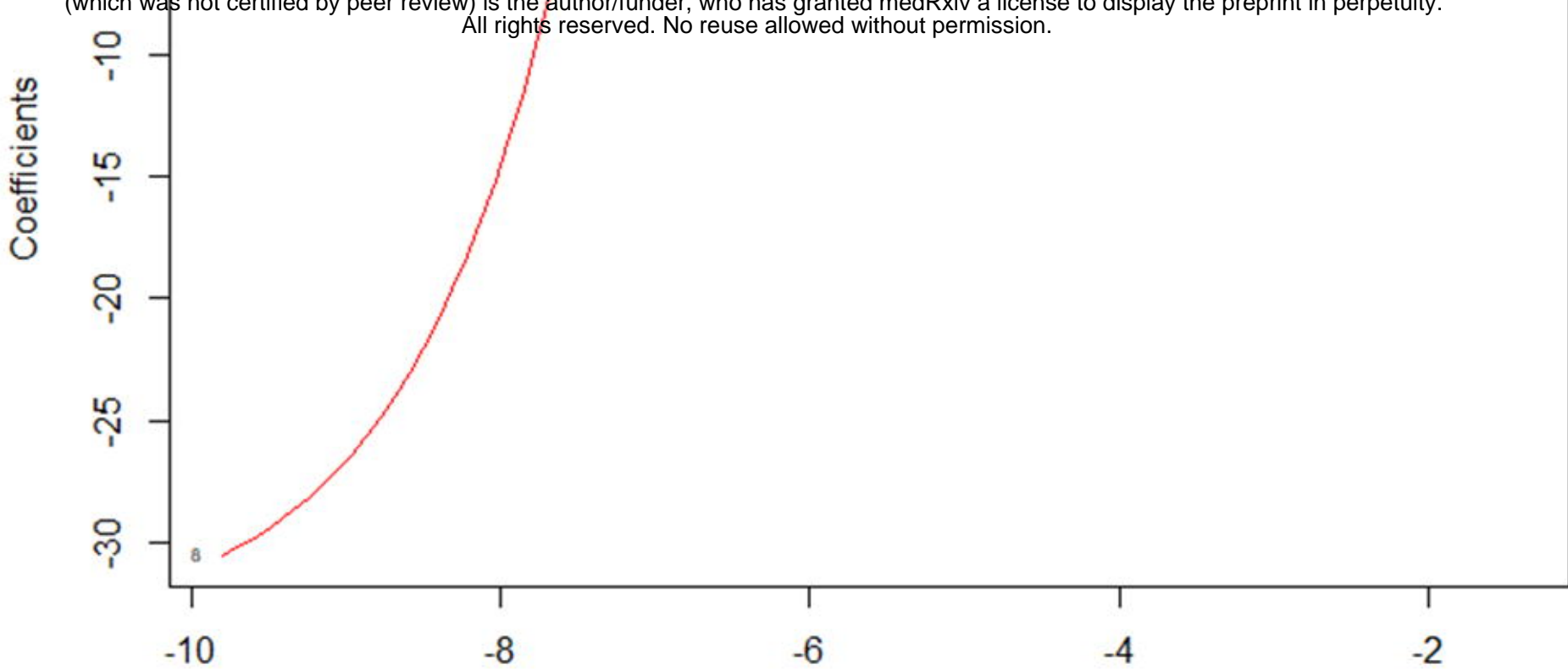

Log Lambda

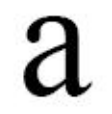

$\begin{array}{lllllllllllllllllll}34 & 35 & 35 & 35 & 34 & 33 & 33 & 29 & 26 & 23 & 22 & 17 & 16 & 11 & 8 & 5 & 1 & 1 & 0\end{array}$

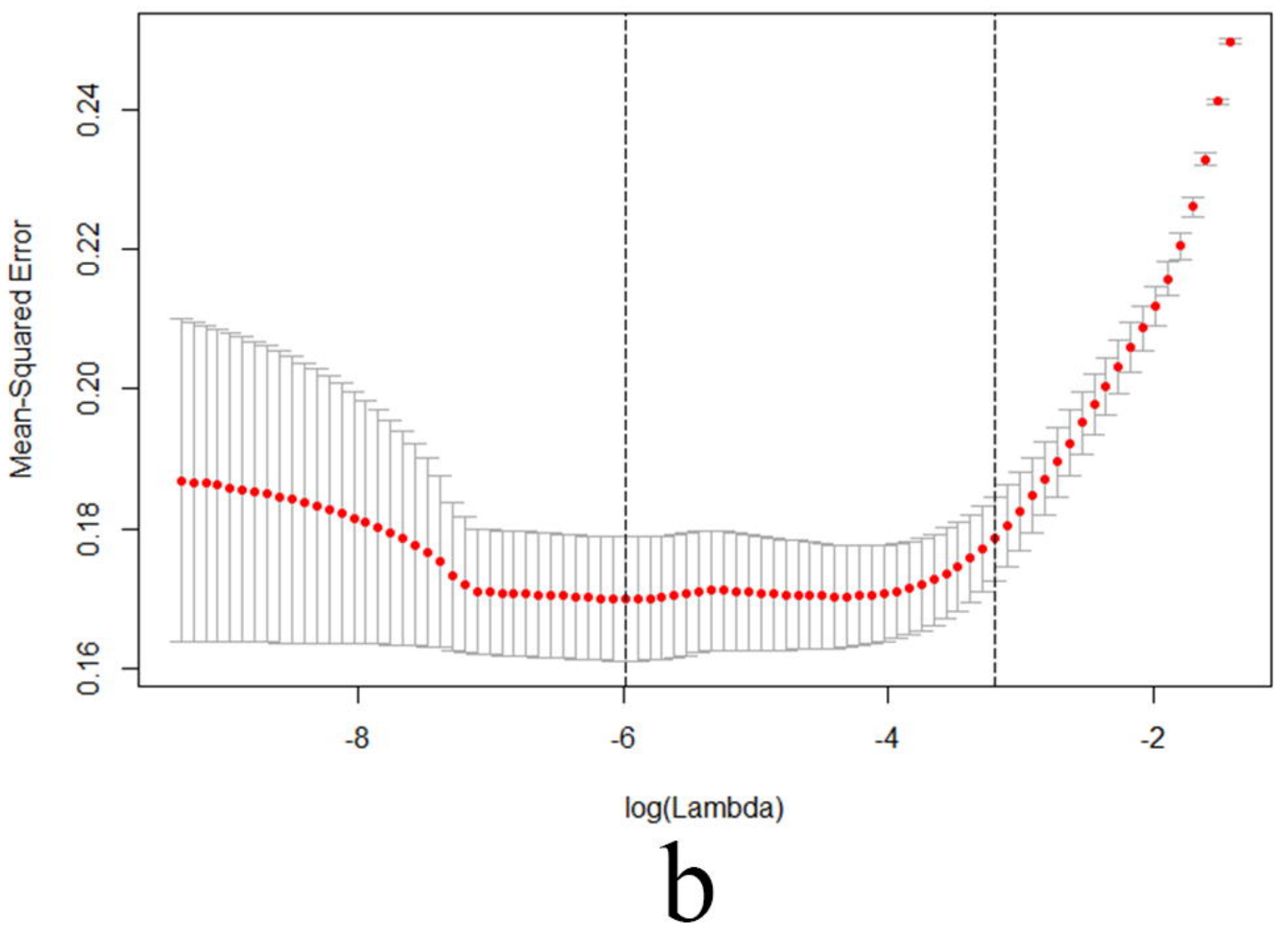




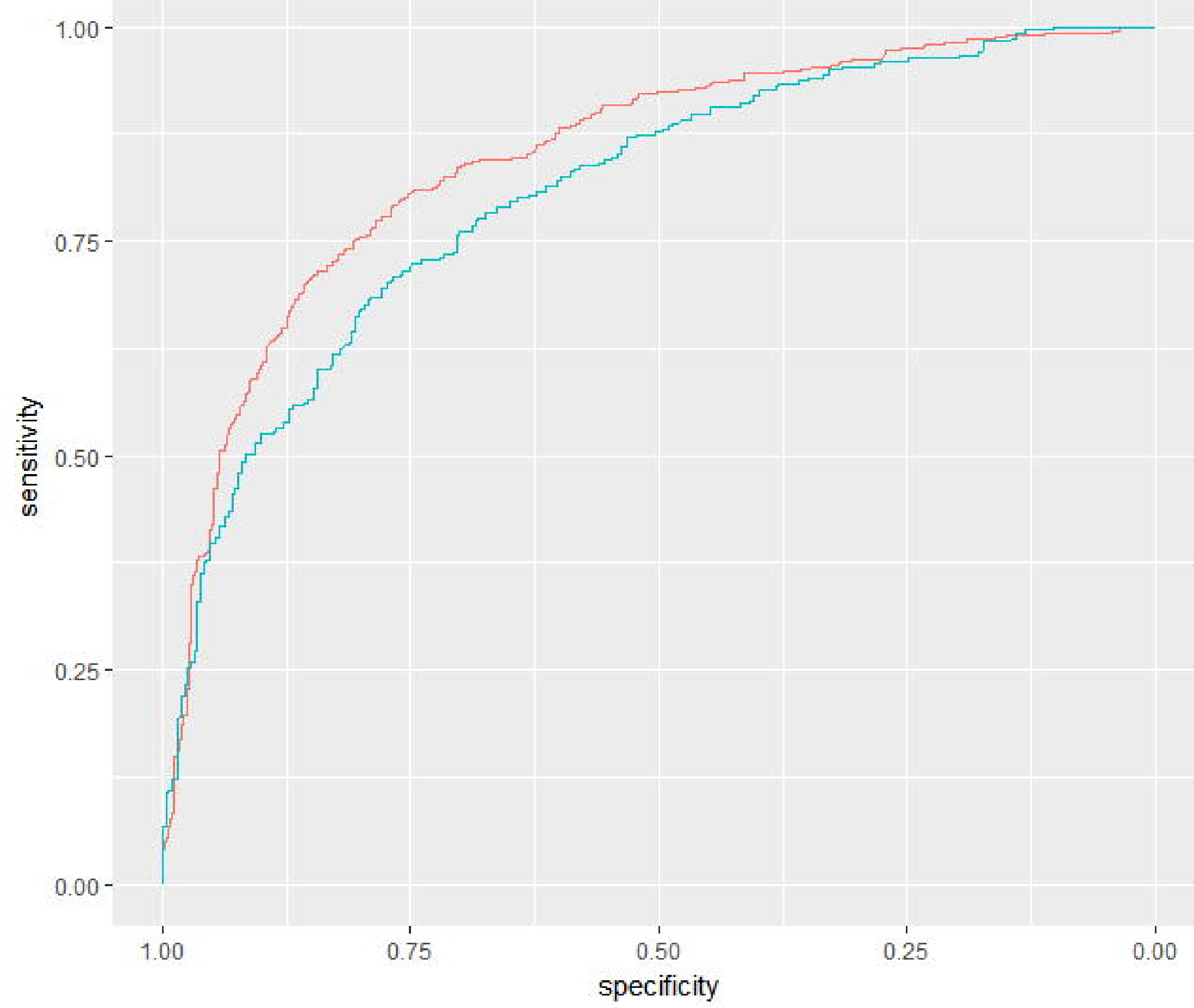

name

- devmodel

- vadmodel 


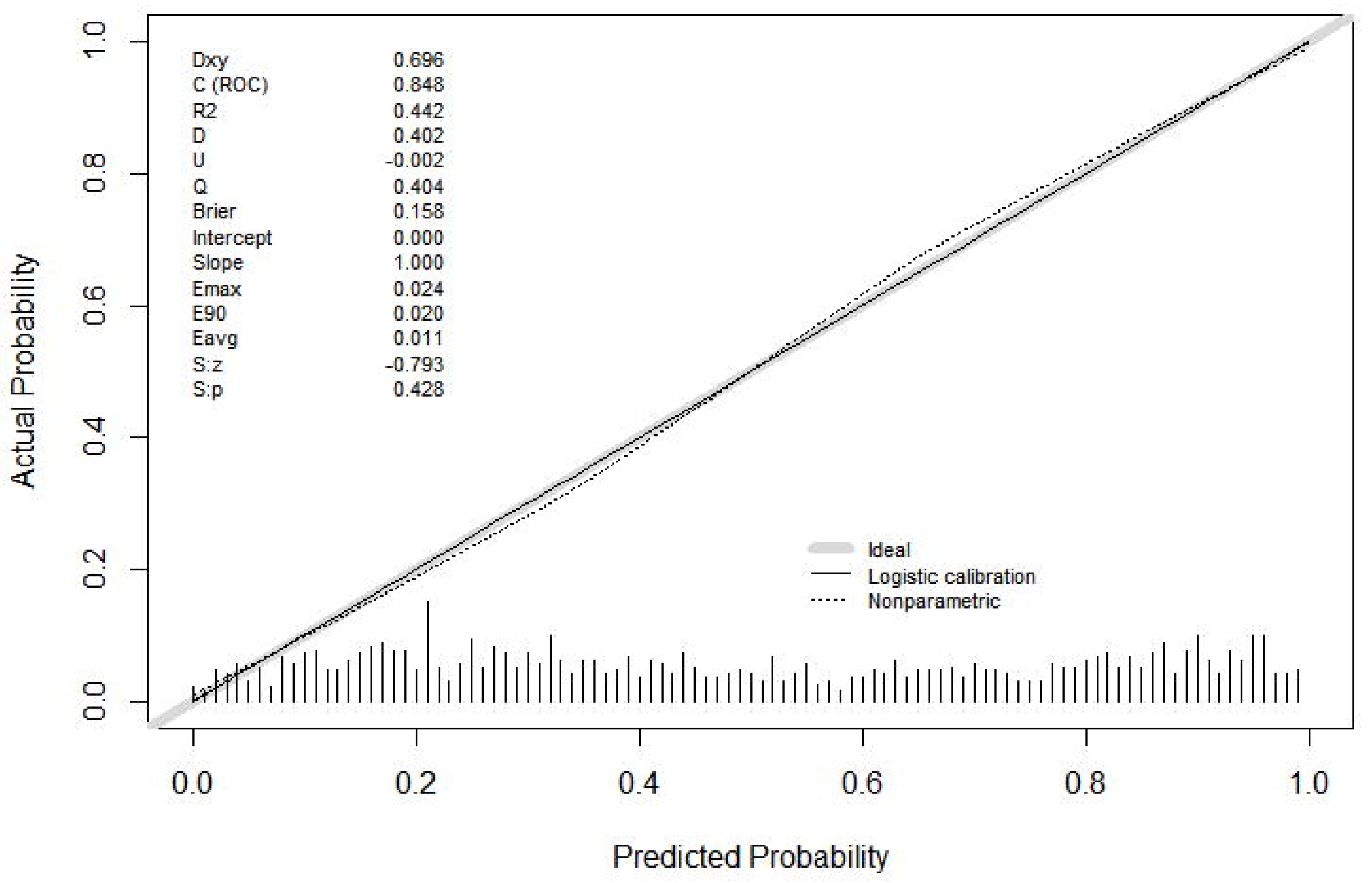




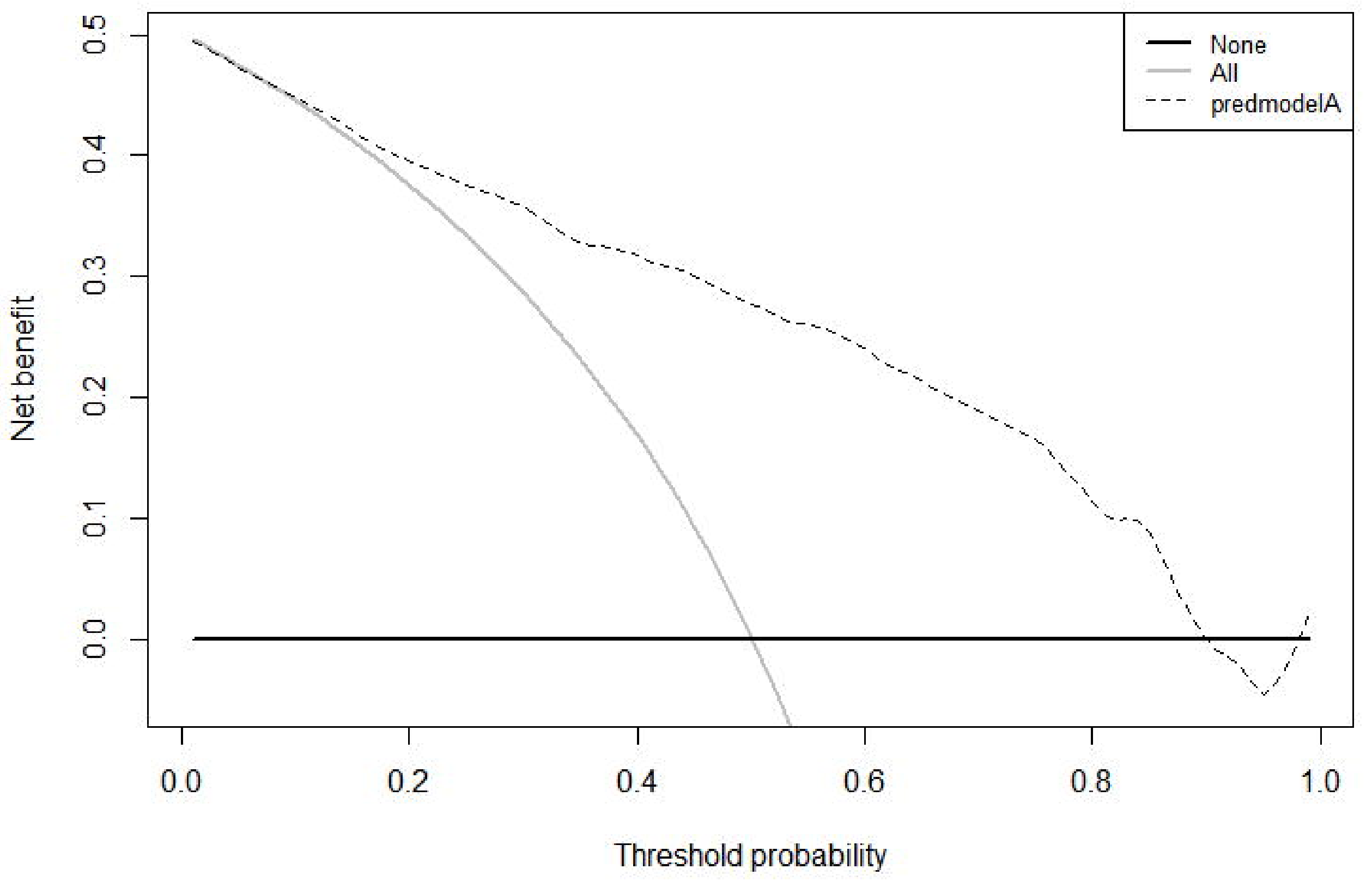




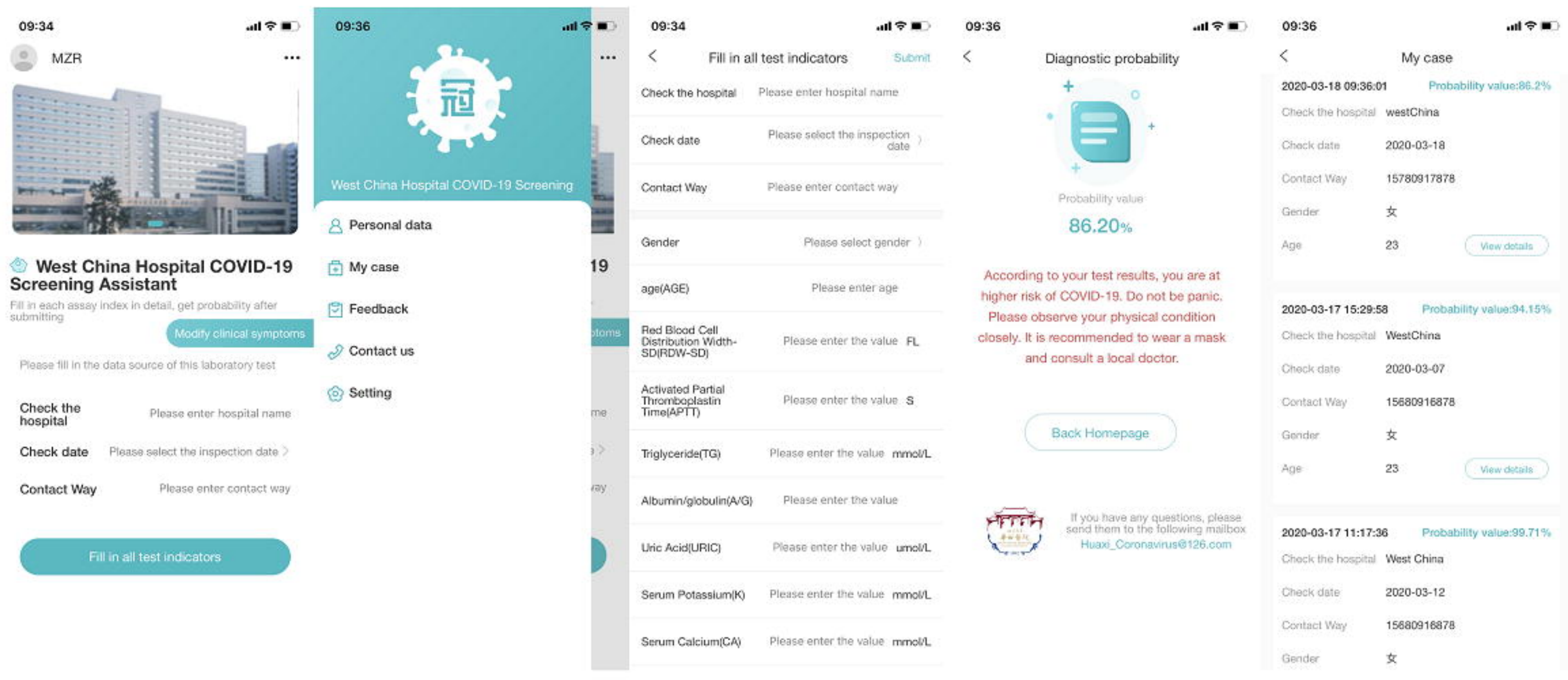

